Journal of

\section{Synchrotron}

\section{Radiation}

ISSN 1600-5775

Received 31 December 2013

Accepted 1 August 2014

\title{
Solvent minimization induces preferential orientation and crystal clustering in serial micro-crystallography on micro-meshes, in situ plates and on a movable crystal conveyor belt
}

\author{
Alexei S. Soares, ${ }^{\text {a* Jeffrey D. Mullen, }}{ }^{\text {b,c }}$ Ruchi M. Parekh, ${ }^{\text {b,d }}$ Grace S. McCarthy, ${ }^{a}$ \\ Christian G. Roessler, ${ }^{a}$ Rick Jackimowicz, ${ }^{a}$ John M. Skinner, ${ }^{a}$ Allen M. Orville, ${ }^{\text {a,e }}$ \\ Marc Allaire $^{\mathrm{a}}$ and Robert M. Sweet ${ }^{\mathrm{a}, \mathrm{e}}$ \\ aPhoton Sciences Directorate, Brookhaven National Laboratory, Upton, NY 11973, USA, 'b Office \\ of Educational Programs, Brookhaven National Laboratory, Upton, NY 11973, USA, 'Physics \\ Department, University of Oregon, Eugene, OR 97403-1274, USA, dSuffolk County Community \\ College, Selden, NY 11784, USA, and ${ }^{\mathbf{e} B i o s c i e n c e s ~ D e p a r t m e n t, ~ B r o o k h a v e n ~ N a t i o n a l ~ L a b o r a t o r y, ~}$ \\ Upton, NY 11973, USA. *E-mail: soares@bnl.gov
}

\begin{abstract}
X-ray diffraction data were obtained at the National Synchrotron Light Source from insulin and lysozyme crystals that were densely deposited on three types of surfaces suitable for serial micro-crystallography: MiTeGen MicroMeshes ${ }^{\circledR}$, Greiner Bio-One Ltd in situ micro-plates, and a moving kapton crystal conveyor belt that is used to deliver crystals directly into the X-ray beam. $6^{\circ}$ wedges of data were taken from $\sim 100$ crystals mounted on each material, and these individual data sets were merged to form nine complete data sets (six from insulin crystals and three from lysozyme crystals). Insulin crystals have a parallelepiped habit with an extended flat face that preferentially aligned with the mounting surfaces, impacting the data collection strategy and the design of the serial crystallography apparatus. Lysozyme crystals had a cuboidal habit and showed no preferential orientation. Preferential orientation occluded regions of reciprocal space when the X-ray beam was incident normal to the datacollection medium surface, requiring a second pass of data collection with the apparatus inclined away from the orthogonal. In addition, crystals measuring less than $20 \mu \mathrm{m}$ were observed to clump together into clusters of crystals. Clustering required that the X-ray beam be adjusted to match the crystal size to prevent overlapping diffraction patterns. No additional problems were encountered with the serial crystallography strategy of combining small randomly oriented wedges of data from a large number of specimens. Highquality data able to support a realistic molecular replacement solution were readily obtained from both crystal types using all three serial crystallography strategies.
\end{abstract}

Keywords: in situ X-ray data collection; crystallography; acoustic droplet ejection; serial crystallography.

\section{Introduction}

Serial micro-crystallography efforts currently explore diverse strategies for combining data from a large number of crystals that transit the X-ray beam either tumbling freely (Boutet \& Robinson, 2008; Weierstall et al., 2008) or deposited on a surface (Ji et al., 2010; Soares et al., 2011; Roessler et al., 2013). New X-ray sources enable the use of micro-crystals because they can focus down to a few micrometers. In high-throughput fragment screening applications, serial crystal handling can accelerate data collection (Tsai et al., 2013) and micro-crystals can accelerate fragment binding (Cole et al., 2014). Challenging structure determination projects such as viruses, membrane proteins and protein-protein complexes will also benefit because these proteins often yield crystal showers. Radiation damage usually prevents obtaining a complete data set from a single micro-crystal, so contributions from many specimens must frequently be merged. At third-generation micro-beam facilities such as APS-GM/CA, diffraction decays to half-maximum (Henderson, 1990) in a few seconds; future synchrotron improvements will continue to push the capability for virtually instantaneous data collection. For example, the 
exposure limit under a $1 \mu \mathrm{m}$ beam at the National Synchrotron Light Source II (NSLS II) FMX beamline is expected to be $5 \mathrm{~ms}$ (Hodgson et al., 2009).

One ubiquitous feature affecting the ultimate viability of serial crystallography strategies is the minimum size that is desirable for specimens in real space and for wedges of data in reciprocal space. Diffracting power is directly proportional to crystal volume so that it is possible to determine the minimum feasible size $\left[19.5 \mu \mathrm{m}\right.$ for insulin, 4:4:1 habit, $\langle I / \sigma(I)\rangle_{\text {last shell }}=2$, $1.8 \AA$ A ; Holton \& Frankel, 2010] needed for a single specimen to yield full data before it is dimmed by radiation damage (Teng \& Moffat, 2002). This minimum crystal size can be overcome if complete data is assembled from multiple crystals. However, merging a very large number of tiny data wedges presents its own challenges.

Most of the serial crystallography strategies that our group is currently exploring require that crystals be deposited onto a two-dimensional surface (flat micro-meshes, planar well bottoms on in situ micro-plates, and a horizontal conveyor belt). Crystal clustering problems can occur when crystals are deposited onto such flat data-collection media with minimal solvent (solvent minimization is required for optimal signalto-noise). Electron microscopists are familiar with the tendency of small objects to cluster in thin water films due to surface tension, making it difficult to image each specimen individually. As very small protein crystals are increasingly used for X-ray crystallography it seems likely that they will aggregate in a similar way. Clean single-crystal diffraction data will require either probing the sample with a tiny beam that is small compared with most crystal-to-crystal distances, or preventing clustering using some physical method akin to electron microscopy grids (Kubalek et al., 1991). Alternatively, software improvements may increase the robustness of data reduction to handle overlapping diffraction from crystal clusters. In addition to clustering, solvent minimization may induce crystals with flat sides to preferentially orient relative to the data-collection media, and data-collection strategies must be adjusted to measure all data.

There is a pressing need to determine the requirements of serial micro-crystallography efforts so that the characteristics of mini-beam and micro-beam X-ray facilities can be chosen to match. The Protein Crystallography Research Resource (PxRR) is operating the mini-beam facility at NSLS-X25 and simultaneously participating in the construction of two microbeam facilities at NSLS-II. Faced with the inevitable trade-offs between different capabilities, we were motivated to begin finding answers to some of these questions. Our group has successfully demonstrated unique new approaches to serial micro-crystallography, such as acoustic droplet ejection (Ellson et al., 2003), which uses sound waves to move crystals directly from their growth trays onto data-collection media (Soares et al., 2011; Cuttitta et al., 2014; Roessler et al., 2013) or to grow protein crystals on plates (Villaseñor et al., 2012) or directly on micro-meshes (Yin et al., 2014). Initially, our serial crystallography efforts were frustrated by difficulties such as preferential orientation and crystal clustering. Here we explore the advantages and challenges of assembling a complete data set from a large number of micro-crystals that are serially presented to the X-ray beam on micro-meshes, in in situ micro-plates, and on a kapton crystal conveyor belt.

\section{Methods}

Serial crystallography is expected to generate a large volume of data that is best handled by data acquisition software that is closely integrated with a database and with image handling software. Our strategy was to identify crystals on flat datacollection media using standard X-ray grid scan techniques (Héroux et al., 2014). Our grid scanning effort was deployed within our existing CBASS beamline control architecture, with built-in data triage using distl (Zhang et al., 2006), data processing functionality using EDNA (Incardona et al., 2009) and access to all output files and metadata via our PXDB database (Skinner et al., 2006). Surfaces containing microcrystals were oriented orthogonal to the X-ray beam. Beamline software automatically detected small crystals on micromeshes using standard grid search techniques, and data were obtained with user-selected data collection parameters (Fig. 1a). In situ crystals in crystallization micro-plates (Fig. 1b) and crystals on the conveyor belt (Fig. 1c) were large enough $(50 \mu \mathrm{m})$ that grid scanning was not needed.

\subsection{Specimen preparation}

The diffraction from very small crystals was easily overwhelmed by background noise when mother liquor was not carefully minimized. Most of the crystals were manually transferred onto MiTeGen micro-meshes (a few crystals were acoustically mounted; data not shown ${ }^{\mathbf{1}}$ ). To improve the signal-to-noise and decrease the contribution of the mother liquor to the X-ray background, the solvent around manually mounted crystals was minimized in a humidity chamber using an absorbent material (such as a dental point) to dab dry each micro-mesh from the bottom. A video of this procedure is available through our YouTube channel (http://www.youtube. com/channel/UCtCiMjlzBnq5VYZzrEi3EiQ). To demonstrate the importance of solvent minimization, we used MicroRT $^{\circledR}$ X-ray Capillaries (MiTeGen) to obtain roomtemperature X-ray diffraction data from the same thermolysin crystal both before and after solvent minimization. The capillary sheath was then removed and the crystal was cooled in liquid nitrogen for $\mathrm{X}$-ray data acquisition at cryogenic temperatures. Thermolysin was used for this experiment because it crystallizes into a rod-shaped habit that is readily partitioned into three X-ray targets (one before solvent minimization, one after solvent minimization, and one at

\footnotetext{
${ }^{1}$ Some crystals were mounted from their crystallization plates onto MiTeGen micro-meshes using an Echo 550 liquid-handling instrument (Labcyte Inc., Sunnyvale, CA, USA). The Echo 550 transfers crystals enveloped in a thin $2.5 \mathrm{~nL}$ blanket of mother liquid (this small volume of solvent minimizes the $\mathrm{X}$-ray background). We postulated that acoustically mounted crystals might have the advantage of low solvent (reduced background noise) without the disadvantages (preferential orientation described in $\$ 3.2$ and crystal clustering described in \$3.3). However, we observed that acoustically mounted specimens clustered together despite low mounting volumes.
} 


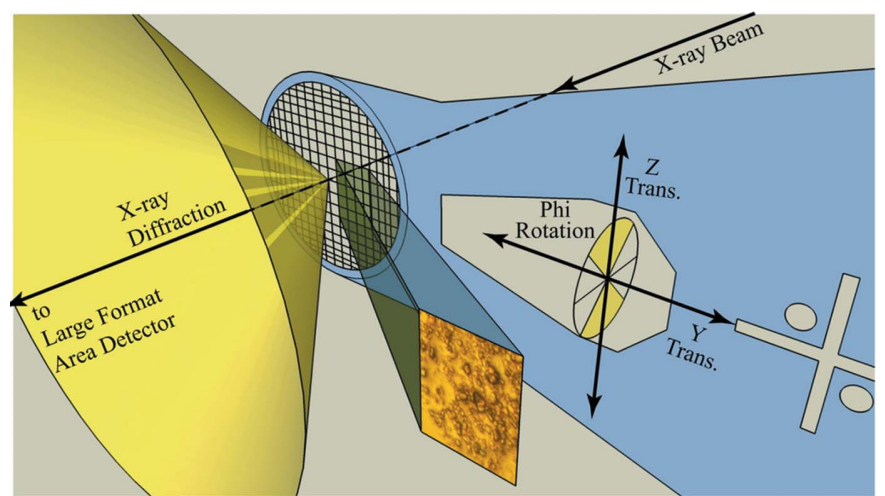

(a)

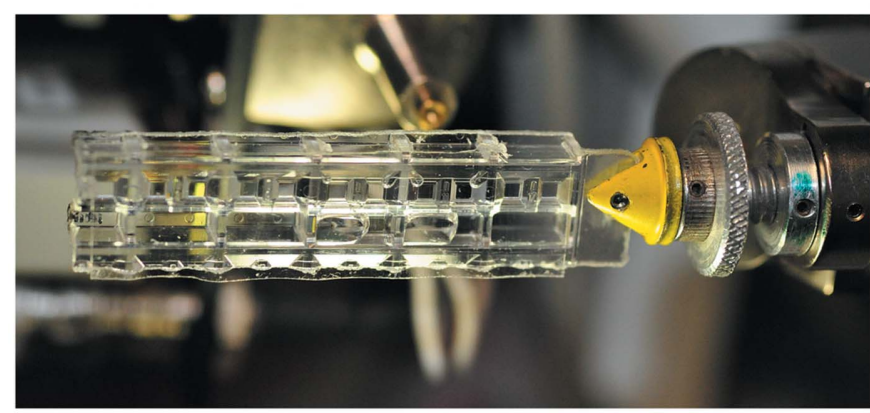

(b)

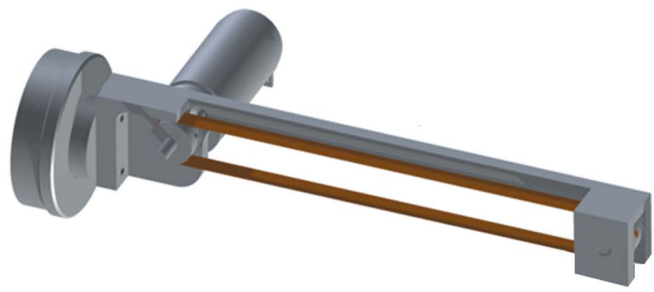

(c)

\section{Figure 1}

Data-collection strategy. The X-ray beam was used to scan for crystals using standard grid-scan techniques. When crystals were found, a $6^{\circ}$ wedge of data was obtained (either a single $6^{\circ}$ rotation or three $2^{\circ}$ rotations). Serial data were obtained from crystals deposited on micromeshes $(a)$, on in situ micro-plates $(b)$ and on a moving conveyor belt system (c) (Roessler et al., 2013). In each case, diffraction from $\sim 100$ individual crystals was collated into a high-quality data set. Data analysis was aided by triaging software. Initially, all data were obtained with the X-ray beam normal to the flat surface of the media on which crystals were deposited. Additional data were obtained with the X-ray beam tilted 30$60^{\circ}$ relative to the flat crystal-containing surface (the additional data were needed because preferential orientation of the crystallographic $c$ axis limited the maximum obtainable completeness using this strategy to $\sim 70 \%$, as described in $\$ 3.2$ ).

$100 \mathrm{~K})$. The data were greatly improved by the solvent minimization procedure (see $\$ 3.1$ ).

Removal of the mother liquor is crucial for optimizing the signal-to-noise of X-ray diffraction data. However, solvent minimization can also cause undesired side effects. One potential complication that can occur during solvent minimization is that the flat crystal faces may interact either with the surface of the data-collection medium (which may cause the crystallographic axis to always point the same way) or with neighboring crystals (which may cause the crystals to cluster into groups). The effect of these attractive forces was observed
Table 1

Nucleation frequency for insulin is proportional to the cool down rate from $T_{1}^{\text {soluble }}=323$ to $T_{2}{ }^{\text {crystal }}=293 \mathrm{~K}$

Careful temperature control yields highly mono-disperse crystal sizes. Sizes were determined by inspection of high-resolution pictures.

\begin{tabular}{lllllc}
\hline Cool down & $\begin{array}{l}\text { Number } \\
\text { measured }\end{array}$ & $\begin{array}{l}\text { Average } \\
\text { size } \\
(\mu \mathrm{m})\end{array}$ & $\begin{array}{l}\text { Standard } \\
\text { deviation } \\
(\mu \mathrm{m})\end{array}$ & $\begin{array}{l}\text { Smallest } \\
\text { size } \\
(\mu \mathrm{m})\end{array}$ & $\begin{array}{l}\text { Largest } \\
\text { size } \\
(\mu \mathrm{m})\end{array}$ \\
\hline$T_{1} \rightarrow T_{2}<10 \mathrm{~s}$ & 255 & 5.2 & 1.5 & 3 & 12 \\
$T_{1} \rightarrow T_{2} 1 \mathrm{~min}$ & 159 & 9.7 & 3.5 & 5 & 27 \\
$T_{1} \rightarrow T_{2} 10 \mathrm{~min}$ & 89 & 21.6 & 7.9 & 8 & 53 \\
$T_{1} \rightarrow T_{2} 120 \mathrm{~min}$ & 74 & 47.3 & 14.9 & 22 & 103 \\
\hline
\end{tabular}

to depend on the shape and size of the protein crystals. We used crystals of insulin and lysozyme, which have very different crystal habits:

Insulin: Flat rhombohedral insulin crystals were grown by dissolving $0.025 \mathrm{~g}$ Sus scrofa (pig) insulin in crystallizing solution $(2.0 \mathrm{ml} 0.02 \mathrm{M} \mathrm{HCl}, 1.0 \mathrm{ml} 0.20 \mathrm{M}$ sodium citrate, $0.6 \mathrm{ml}$ acetone, $0.2 \mathrm{ml} \mathrm{H}_{2} \mathrm{O}$ and $0.2 \mathrm{ml} 0.12 M \mathrm{ZnSO}_{4}$ ) at $T_{1}$ soluble $=323 \mathrm{~K}$ and allowing the solution to cool slowly to $T_{2}{ }^{\text {crystal }}=293 \mathrm{~K}$. The cooling rate determines the growth size for crystals (Table 1). Crystals of all sizes form a rhomboid with side length ratios of 4:4:1 (Fig. 2). By carefully controlling the cooling rate, our crystals were highly monodisperse with a side length variance of $40 \%$ of the mean (Soares et al., 2003).

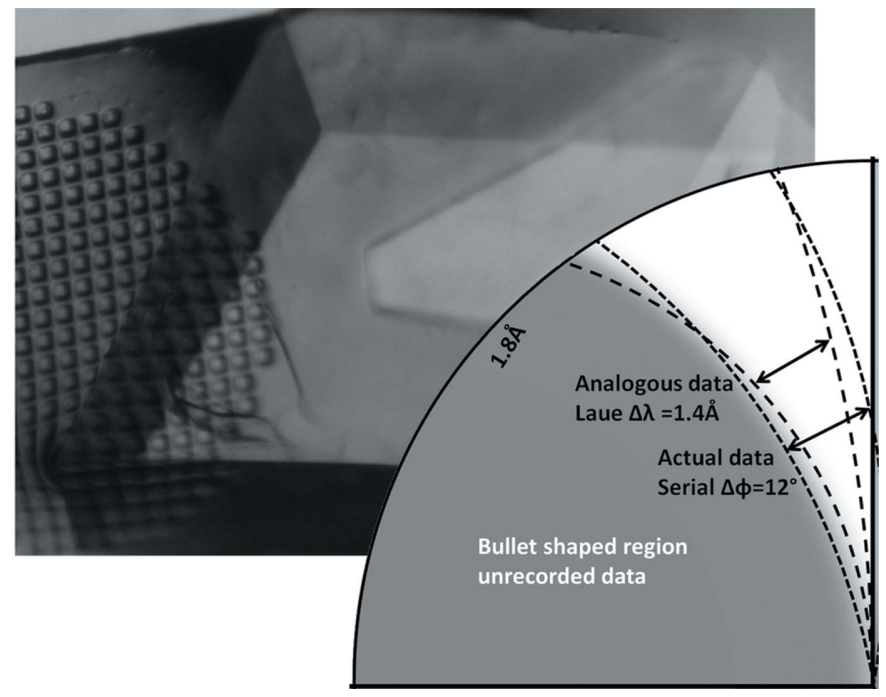

Figure 2

Strongly diffracting insulin crystals with side length ratios of $\sim 4: 4: 1$ are easily grown over a very large range between $2000 \mu \mathrm{m}$ and $4 \mu \mathrm{m}$. A view of one $300 \mu \mathrm{m}$ crystal is shown here. The flat rhomboid face of these crystals frequently rests against the supporting micro-mesh, orienting the crystallographic $c$ axis nearly parallel to the X-ray beam. Although the 5,10 and $20 \mu \mathrm{m}$ specimens used in this study were too small to orient visually, the diffraction data confirms a significant bullet-shaped region of un-measurable nodes adjacent to the $c$ axis (inset). Flat insulin crystals preferentially orient with the $c$ axis orthogonal to the support (i.e. into the $\mathrm{X}$-ray beam). $6^{\circ}$ librations from $100+$ reciprocal space wedges all around the $c$ axis guarantee that the volume of measurable data (dotted line) retains constant thickness around the X-ray beam. However, a bulletshaped region of reciprocal space remains occluded (shaded region). The situation is analogous to a Laue case with $\lambda_{\min } \simeq 0.6 \AA$ and $\lambda_{\max } \simeq 2.0 \AA$ (dashed line). Fortunately, the missing data were readily available by tilting the micro-mesh about omega prior to the grid scan. 
Crystals were cryo-protected using $1.2 \mathrm{M}$ trehalose or $30 \% \mathrm{v} / \mathrm{v}$ ethylene glycol. Specimens were deposited in a thin layer on micro-meshes, in situ micro-plates and on a movable crystal conveyor belt (Fig. 1).

Lysozyme: Cuboidal lysozyme crystals measuring $50 \mu \mathrm{m}$ on each side were obtained by batch crystallization $\left(120 \mathrm{mg} \mathrm{ml}^{-1}\right.$ lysozyme dissolved in $0.1 M \mathrm{NaAc}, \mathrm{pH} 4.6$ and $10 \%$ ethylene glycol, with $4 \% \mathrm{NaCl}$ precipitant). Crystals were cryoprotected using $20 \%$ glycerol.

\subsection{Data collection}

Serial crystallography data were obtained from crystals that resided on three types of data-collection media:

(i) On MiTeGen MicroMeshes ${ }^{(i i}$ (Fig. 1a). Crystals mounted on micro-meshes had sizes between $5 \mu \mathrm{m}$ and $50 \mu \mathrm{m}$. Data were obtained using a conventional goniometer.

(ii) On Greiner Bio-One ${ }^{\circledR}$ micro-plates (Fig. 1b). Plate data were obtained in situ from $\sim 50 \mu \mathrm{m}$ crystals using a robotic G-Rob plate handler (NatX-ray LLC, San Diego, CA, USA) or using a conventional goniometer (Axford et al., 2012). Goniometer-mounted plates were cut into 16 pieces, each of which was attached to a magnetic mount so that data could be obtained using a conventional goniometer.

(iii) On a movable conveyor belt system (Fig. 1c). Data were obtained from crystals that were serially transferred to a goniometer-mounted conveyor belt system (Roessler et al., 2013).

All of our small $(<50 \mu \mathrm{m})$ specimens were serially interrogated by partitioning each crystal containing micro-mesh into $20 \mu \mathrm{m} \times 20 \mu \mathrm{m}$ fields and scanning each field using the $\mathrm{X}$-ray beam. Data were obtained from crystal-containing areas using either one $6^{\circ}$ rotation or three $2^{\circ}$ rotations (the beam size was matched to the expected crystal size). The unusually wide $6^{\circ}$ data wedges were necessary so that the data from one crystal had sufficient reflections in common with the data from the next crystal for scaling ${ }^{2}$. Using much smaller wedges of data $\left(>1^{\circ}\right)$ would be feasible if the image-to-image matching process was automated, but the time needed to manually sort through thousands of single exposures to search for fortuitously oriented next-in-line images proved prohibitive. This scaling problem is mitigated when larger regions of reciprocal space are recorded from each micro-crystal.

Our specimens were exposed for a prolonged time interval to ensure that each $6^{\circ}$ data wedge had a high $I / \sigma(I)$. At X25, the nominal $2 \times 10^{11}$ photons s$^{-1}$ flux through $100 \mu \mathrm{m}^{2}$ was $x-y$ slit-shaped to form a mini-beam as small as $20 \mu \mathrm{m}^{2}$. The flux density translates into $\sim 180 \mathrm{~s}$ needed to reach the Henderson dose limit $\left(D_{1 / 4}=10^{7} \mathrm{~Gy}\right)$, or $30 \mathrm{~s}$ per degree for a $6^{\circ}$ wedge. A second data-collection pass with the micro-mesh

\footnotetext{
2 The strategy of recording a $6^{\circ}$ wedge of data from each crystal (three images of $2^{\circ}$ width) is not optimal for most purposes. In this study the exaggerated frame width was selected for two reasons. First, it is much more difficult to merge narrow wedges of data, particularly when the space group is polar. Second, at NSLS II the conveyor belt will deliver crystals to the very bright AMX X-ray beam very rapidly and the $20 \mathrm{~Hz}$ framing rate for the Pilatus $6 \mathrm{M}$ detector will not be able to keep up. For this reason we are interested in exploring how much of a data quality penalty is caused by measuring unusually wide rotations.
}

rotated by $30-60^{\circ}$ was sometimes needed (see $\$ 3.2$ ), and the exposure time was reduced to allow for the additional datacollection sweep.

\subsection{Merging data}

For insulin crystals, the $R 3$ space group is polar so that reindexing was frequently needed to stitch together the $6^{\circ}$ data wedges from multiple crystals using Scalepack (Otwinowski \& Minor, 2001). Our strategy was to pool a handful of compatible reduced images (about a dozen) by trial and error. Success required the coincidental presence of a sufficient number of simultaneously recorded reflections for each image to scale to the others. These scaled images then served as a 'keystone' to assemble each additional inbound image. Merging data from $\sim 100$ crystals was straightforward when $3 \times 2^{\circ}$ contiguous wedges were measured. HKL2000 had no difficulty indexing, scaling or merging each triad. Dealing with single $6^{\circ}$ rotations was slightly more problematic (uncertainty in the crystal mosaicity impeded merging images that only shared a handful of simultaneously recorded reflections, so the 'keystone' images were more difficult to assemble). A more robust strategy for merging data in polar space groups was recently described (Brehm \& Diederichs, 2014).

For comparison, data from differently sized crystals were segregated. For example, $10 \mu \mathrm{m}$ and $20 \mu \mathrm{m}$ crystals were separated. The high $R_{\text {merge }}$ values are a consequence of merging data from $\sim 100$ individual crystals into a single data set (Diederichs \& Karplus, 1997). Relatively high $R_{\text {merge }}$ values were tolerated by the structure determination software that we used and these initially high- $R_{\text {merge }}$ values became increasingly insignificant as each data set supported the determination of a high-quality coordinate file. All six data sets supported a molecular-replacement solution using MOLREP (Vagin \& Teplyakov, 1997) with a polyalanine search model (3rto and 1lyz, all residues changed to Ala) (Diamond, 1974). Each structure was completed and refined using ARP/wARP and REFMAC (Langer et al., 2008; Murshudov et al., 2011). Parameters for data collection, structure solution and refinement are shown in Table 2 for all six data sets from insulin crystals. The three data sets from lysozyme crystals are not shown. Other than completeness differences discussed later, there were no significant differences between the quality of data obtained from serial data collection using micro-meshes, in situ plates and the movable conveyor belt system.

\section{Results}

Data from small crystals mounted on micro-meshes were obtained at NSLS-X12b from $20 \mu \mathrm{m}$ porcine insulin crystals in space group $R 3$ scanned by a $30 \mu \mathrm{m} \times 30 \mu \mathrm{m}$ beam, and at NSLS-X25 from $10 \mu \mathrm{m}$ and $5 \mu \mathrm{m}$ porcine insulin crystals scanned by a $20 \mu \mathrm{m} \times 20 \mu \mathrm{m}$ beam (Table 2 ). Data from larger lysozyme and insulin crystals $(>50 \mu \mathrm{m})$ were obtained at NSLS-X25 (micro-meshes), NSLS-X29 (in situ plates) and NSLS-X12c (conveyor belt). Usable data sets were assembled 
Table 2

Data were obtained from $5 \mu \mathrm{m}, 10 \mu \mathrm{m}, 20 \mu \mathrm{m}$ and $50 \mu \mathrm{m}$ insulin crystals (shown below) as well as from lysozyme crystals (not shown).

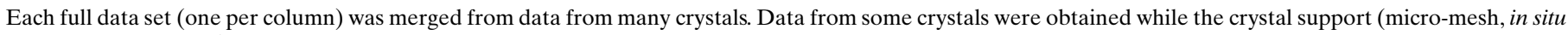

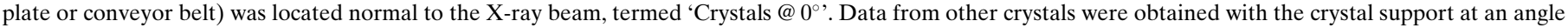

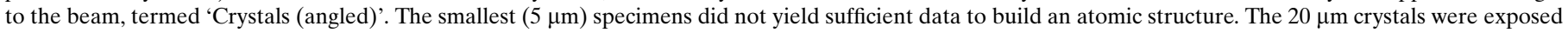

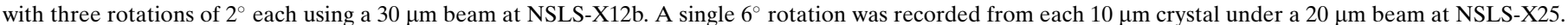

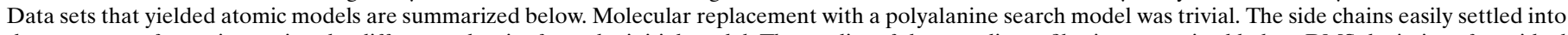

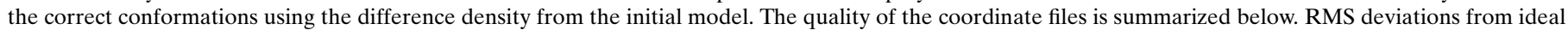
for the final structures are shown.

\begin{tabular}{|c|c|c|c|c|c|c|}
\hline & Mesh & Mesh & Mesh & Mesh & Plate & Belt \\
\hline Crystal size $(\mu \mathrm{m})$ & 10 & 20 & 20 & 50 & 50 & 50 \\
\hline X-ray source & $\mathrm{X} 25$ & $\mathrm{X} 12 \mathrm{~b}$ & $\mathrm{X} 12 \mathrm{~b}$ & $\mathrm{X} 25$ & $\mathrm{X} 29$ & $\mathrm{X} 12 \mathrm{c}$ \\
\hline Beam size $(\mu \mathrm{m})$ & 20 & 30 & 30 & 50 & 100 & 200 \\
\hline Surface & Mesh & Mesh & Mesh & Mesh & Plate & Belt \\
\hline Strategy & $1 \times 6^{\circ}$ & $1 \times 6^{\circ}$ & $3 \times 2^{\circ}$ & $3 \times 2^{\circ}$ & $3 \times 2^{\circ}$ & $3 \times 2^{\circ}$ \\
\hline Resolution (̊̊) & 1.7 & 1.9 & 1.9 & 1.8 & 1.8 & 1.8 \\
\hline Crystal number & 101 & 114 & 192 & 88 & 88 & 88 \\
\hline Crystals@ $0^{\circ}$ & 66 & 95 & 118 & 55 & 55 & 55 \\
\hline Completeness (\%) & 79.3 & 81.4 & 81.9 & 80.8 & 68.9 & 67.1 \\
\hline Limit (\%) & 82.5 & 87.0 & 86.3 & 90.0 & 76.1 & 70.6 \\
\hline Crystals (angled) & 35 & 19 & 74 & 33 & 33 & 33 \\
\hline Angle to beam & $60^{\circ}$ & $60^{\circ}$ & $60^{\circ}$ & $45^{\circ}$ & $30^{\circ}$ & $45^{\circ}$ \\
\hline Completeness (\%) & 93.4 & 89.8 & 96.3 & 98.1 & 91.8 & 97.4 \\
\hline Limit (\%) & 100.0 & 100.0 & 100.0 & 100.0 & 99.7 & 100.0 \\
\hline$R_{\text {merge }}(\%)$ & $15.0(71.2)$ & $14.2(59.7)$ & $9.8(51.2)$ & $20.6(99.2)$ & $29.9(92.9)$ & $26.3(61.7)$ \\
\hline$I / \sigma(I)$ & $19.8(1.3)$ & $16.5(1.0)$ & $14.5(1.9)$ & $18.0(1.0)$ & $18.2(1.0)$ & $13.4(2.9)$ \\
\hline Multiplicity & 4.6 & 7.8 & 14.2 & 4.5 & 12.5 & 5.4 \\
\hline No. of reflections & 8747 & 5881 & 6307 & 7736 & 7241 & 7681 \\
\hline$R_{\text {initial }}(\%)$ & 41.4 & 44.2 & 39.8 & 43.3 & 39.5 & 39.6 \\
\hline$R_{\text {work }}(\%)$ & 26.6 & 22.3 & 22.3 & 21.2 & 21.0 & 25.9 \\
\hline$R_{\text {free }}(\%)$ & 28.5 & 25.2 & 27.7 & 28.0 & 26.3 & 33.0 \\
\hline \multicolumn{7}{|l|}{ RMS deviations } \\
\hline Bond lengths $(\AA)$ & 0.037 & 0.031 & 0.026 & 0.023 & 0.034 & 0.040 \\
\hline Bond angles $\left({ }^{\circ}\right)$ & 2.825 & 2.678 & 2.416 & 2.283 & 2.772 & 3.541 \\
\hline
\end{tabular}

from $6^{\circ}$ reciprocal space wedges measured either with a single $6^{\circ}$ rotation or with three adjacent $2^{\circ}$ rotations, as indicated in the table. Wedges were indexed and scaled using HKL2000 and collated together using Scalepack. $5 \mu \mathrm{m}$ crystals almost always produced overlapping diffraction patterns and the amount of indexable data was insufficient to build an atomic model. Crystals with a longest side length of $10 \mu \mathrm{m}$ or more yielded high-quality structures (Fig. 3). The refined models show no evidence of radiation damage such as broken disulfide bonds. ${ }^{3}$

\subsection{Solvent minimization}

Fig. 4 compares the signal-to-noise ratio $[I / \sigma(I)]$ for roomtemperature X-ray data $\left(0.2 \mathrm{~s}\right.$ per $0.1^{\circ}$ rotation with $97 \%$ attenuation of the X-ray beam) obtained from the same thermolysin crystal before and after the solvent minimization procedure (\$2.1), as well as data from the same crystal after cryo-cooling in liquid nitrogen $\left(2.0 \mathrm{~s}\right.$ per $2.0^{\circ}$ rotation with no attenuation). The solvent minimization greatly improved the quality of the X-ray diffraction data. The resolution limit ( $\mathrm{S} / \mathrm{N}>1.0)$ was improved from 1.91 to $1.77 \AA$. The roomtemperature data after solvent minimization have zero counts observed in most of the background pixels.

\footnotetext{
3 The disulfide bonds in insulin are highly susceptible to bond breakage induced by X-ray radiation (Helliwell, 1988).
}

\subsection{Preferential orientation}

The flat rhomboid habit of our insulin specimens preferentially orients the crystallographic $c$ axis normal to the collection medium surface (Fig. 2). To simplify software code development, we initially scanned our grids with the X-ray beam traversing the flat face at a $90^{\circ}$ angle. This meant that the $c$ axis of the majority of the crystals was approximately parallel to the X-ray beam during the experiment. Although the axis of rotation was perpendicular to the X-ray beam, the $6^{\circ}$ of rotation for each specimen was much less significant than the effect of sampling hundreds of crystals with random rotations around a fixed $c$ axis. Consequently, our initial experiment sampled all possible rotations of crystals with the $c$ axis approximately parallel to the beam, convoluted with a small $6^{\circ}$ libration. This resulted in a very substantial 'bullet-shaped' region of unobtainable data (Table 2). The initial data sets resembled single-crystal Laue stills with $\lambda_{\text {min }} \simeq 0.6 \AA$ and $\lambda_{\text {max }} \simeq 2.0 \AA$ (Fig. 2, inset).

The completeness of each data set asymptotically approached a limit value that was less than $100 \%$. The maximum completeness was calculated using a least-squares fit to equation (1),

$$
C=C_{\max }\left(\frac{n}{n+N}\right),
$$

where $C$ is the observed completeness, $C_{\max }$ is the limit completeness after addition of many data sets, $n$ is the number 

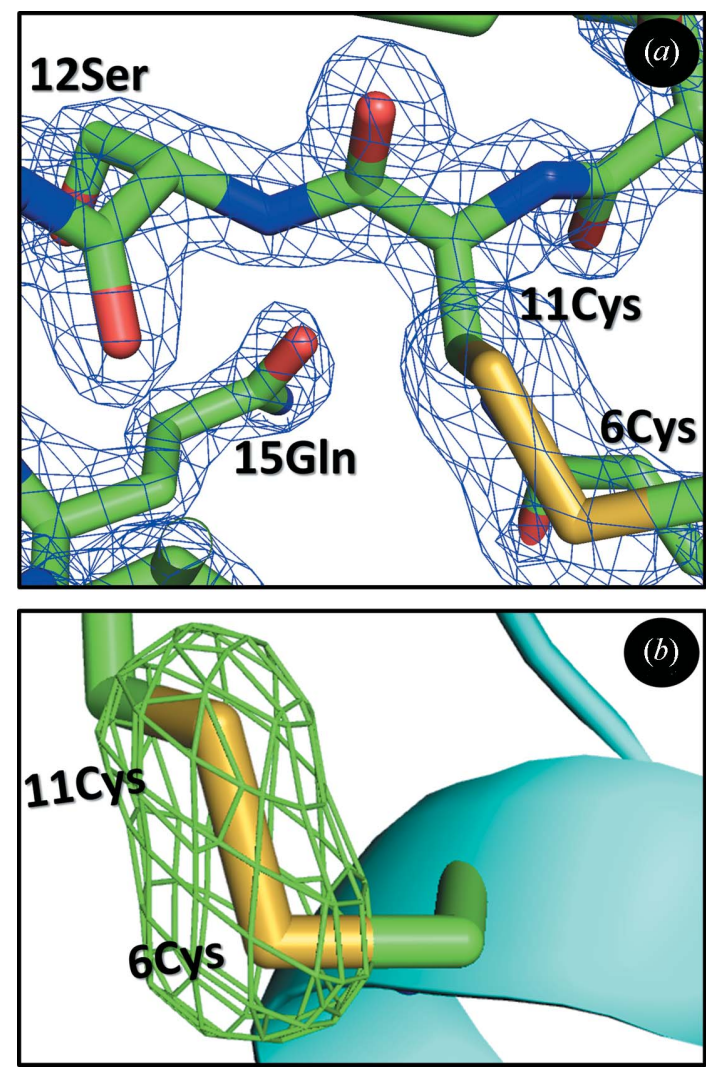

\section{Figure 3}

Crystals with a longest side length of $10 \mu \mathrm{m}$ or more yielded molecularreplacement structures from polyalanine search models. (a) After refinement, the electron density from 192 different $20 \mu \mathrm{m}$ crystals adequately fits the model $\left(R=22.3, R_{\text {free }}=27.7\right)$. The region shown is in the vicinity of 6Cys-11Cys $\left(2 F_{\mathrm{o}}-F_{\mathrm{c}}\right.$ contoured at $\left.3 \sigma\right)$. The disulfide bond shows no signs of breakage, because the high overall radiation dose was spread over many crystals. (b) A close-up view of the same region is shown with the refined model of the in situ plate mounted crystal (data merged from 88 crystals, $R=21.0, R_{\text {free }}=26.3$ ). A difference omit map is contoured at $2 \sigma$ (the two cysteine sulfur atoms were omitted from the model). No evidence of damage to the disulfide bond is visible, even though the data were obtained at room temperature (where radiation damage should have been larger).

of data sets merged, and $N$ is a unit-less fitting parameter. The best values for $C_{\max }$ and $N$ were calculated by least-squares fit. The limit completeness was approximately $90 \%$ when using micro-meshes, and even less for crystals on plates and on the conveyor belt (micro-mesh data have a higher limit completeness because the micro-meshes have a natural curvature which prevents the crystals from being exactly normal to the $\mathrm{X}$-ray beam). To address the problem of limited completeness, data were obtained from a limited number of specimens after rotating the collection media by 30 to $60^{\circ}$ (Fig. 5).

It is likely that the flat shape of insulin crystals plays a role in aligning the crystallographic $c$ axis perpendicular to the surfaces on which the insulin crystals are deposited. In contrast, lysozyme crystals have a cuboidal habit with no extended planar surface. Consequently, lysozyme crystals were not observed to preferentially orient a crystallographic axis (Fig. 5, inset $\alpha$ ).

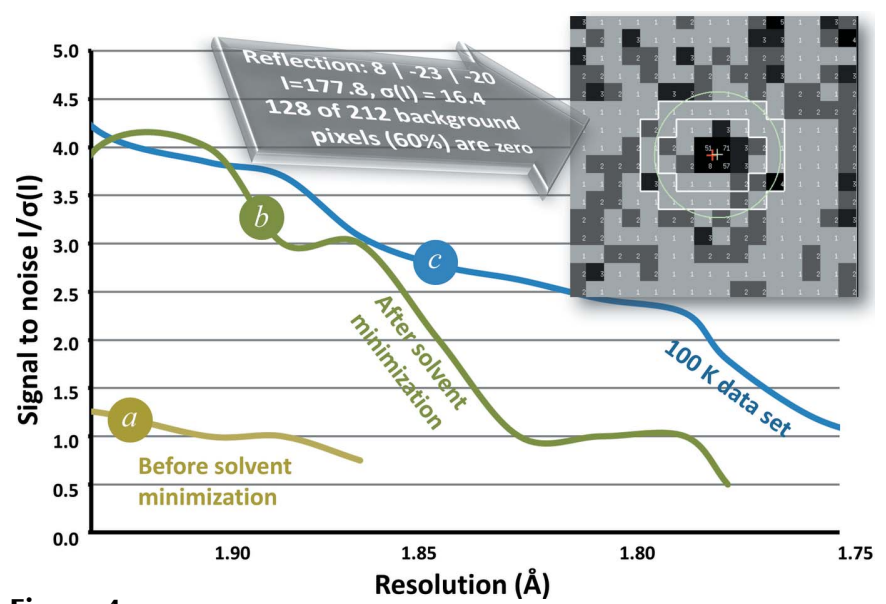

Figure 4

Solvent minimization. The signal-to-noise ratio $[I / \sigma(I)]$ is plotted for the same thermolysin crystal at room temperature before solvent minimization $(a)$, at room temperature after solvent minimization $(b)$ and at $100 \mathrm{~K}$ (c). The room-temperature data sets consisted of $0.1^{\circ}$ rotations with a duration of $0.2 \mathrm{~s}$, with the X-ray beam attenuated by $97 \%$. The $100 \mathrm{~K}$ data set consisted of $2.0^{\circ}$ rotations with a duration of $2.0 \mathrm{~s}$, using the full X-ray beam. The inset shows the $8 \overline{23} \overline{20}$ reflection after solvent minimization. The background integration box for this reflection had exactly zero observed counts for $60 \%$ of the pixels (so that the readout value was equal to the Pilatus $6 \mathrm{M}$ baseline of 1$)$.

\subsection{Crystal clustering}

The $5 \mu \mathrm{m}$ crystals proved extremely difficult to index because virtually every rotation image was either blank or contained too many overlapping diffraction patterns. After examining many such images, we suspected that the distribution of $5 \mu \mathrm{m}$ crystals within each micro-mesh could not be random. Close visual examination of several micro-meshes confirmed that all of our specimens aggregated into clusters during the mother liquor wicking procedure, and that this clustering was more pronounced with small crystals compared with large crystals. Fig. 6 shows one example of small insulin crystals that are highly clustered.

A statistical analysis of the $x, y$ coordinates for 5, 10 and $20 \mu \mathrm{m}$ crystals on separate micro-meshes confirmed this pronounced clustering phenomenon. Fig. 7 shows the normalized frequency ( $5 \mu \mathrm{m}$ average crystal size) of observation for each crystal-to-crystal distance (the distance between crystals was defined as the distance between the crystal centers). The figure shows a pronounced peak at $7 \mu \mathrm{m}$, indicating that small insulin crystals are very likely to be located immediately adjacent to another crystal. The clustering effect occurs over short ranges only, so that larger crystal-to-crystal distances are not affected. We did not determine what force causes crystals to cluster over short distances, but we hypothesize that surface tension may play a role.

Grid scans using a $5 \mu \mathrm{m}$ beam at APS-GM/CA substantially mitigated this specimen overlap problem because when the beam size is comparable with the specimen size the crystals are likely to be illuminated one at a time. Once each crystal in a cluster is indexed with a small beam, it would be feasible to collect simultaneous data on all of them using a larger beam. 


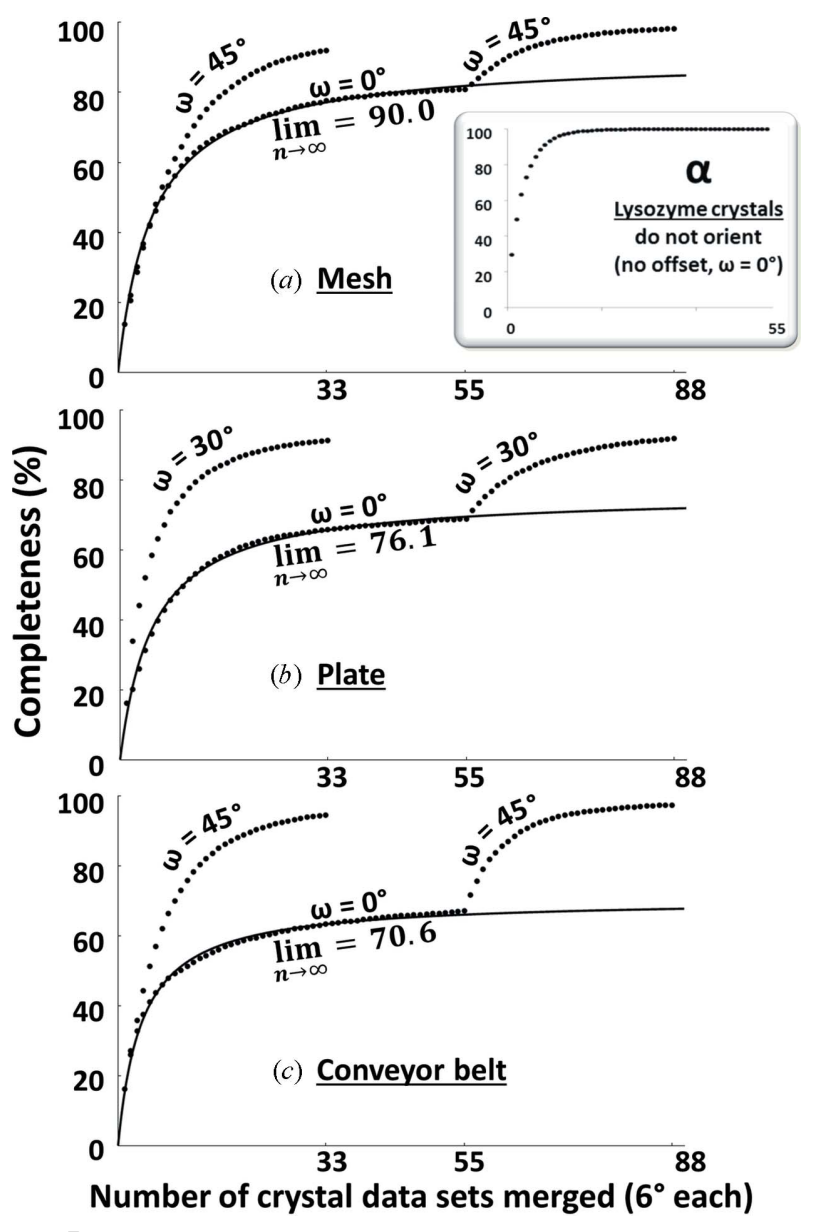

Figure 5

These graphs show completeness of the data from porcine insulin (percent) against the number of $6^{\circ}$ data wedges that were merged together from crystals on micro-meshes $(a)$, on in situ plates $(b)$ and on a movable conveyor belt $(c)$. Two plots are shown in each panel. The lower plot shows completeness as $55 \times 6^{\circ}$ data wedges are merged with the flat surface of the data-collection media normal to the X-ray beam, followed by $33 \times 6^{\circ}$ data wedges with the flat surface offset to the X-ray beam by $30^{\circ}$ (plates) or $45^{\circ}$ (micro-meshes and conveyor belt). In the upper plot the offset data wedges are merged by themselves. An asymptotic best fit shows that, without the offset, the completeness of the data approaches a limit value of less than $100 \%$. In contrast, when offset data are included (either alone or in combination with the 'flat' data), $100 \%$ completeness is achieved. The data were smoothed by merging the $6^{\circ}$ wedges in 100 random permutations, and averaging the results. The inset shows a similar calculation for $6^{\circ}$ wedges of data from 55 lysozyme crystals (panel $\alpha$ ). The lysozyme crystals do not appear to preferentially orient their crystallographic axes relative to the micro-mesh surface. The completeness of the merged data file rapidly approaches $100 \%$ regardless of whether the micro-mesh is presented normal to the X-ray beam or at an angle. The same results were observed with lysozyme crystals in Grainer in situ plates and on the conveyor belt (data not shown).

Software being developed by other groups to de-convolute overlapping diffraction patterns is another alternative solution.

\section{Discussion}

We demonstrate that a workable data set can be assembled from $100+$ narrow reciprocal-space wedges drawn from $6^{\circ}$ rotations of randomly oriented micro-crystals $(10-20 \mu \mathrm{m})$ that

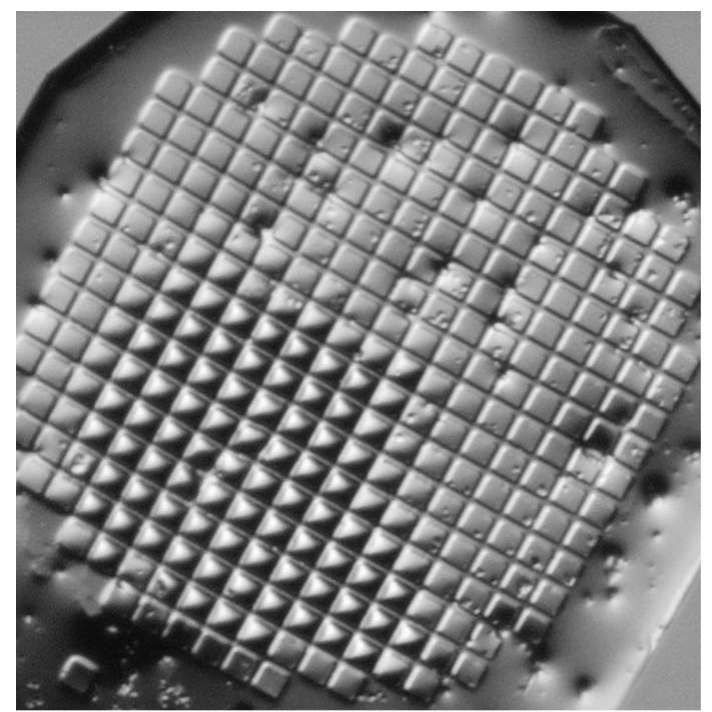

Figure 6

Careful minimization of mother liquor was an indispensable step to observe adequate signal-to-noise in the diffraction pattern. One undesired side effect is that solvent minimization induces crystals to aggregate into tight clusters of crystals that yield overlapping diffraction patterns. The $5 \mu \mathrm{m}$ insulin crystals shown here diffracted to $2.3 \AA$, but no structure was forthcoming because of persistent overlap problems. Realistic molecular-replacement structures were readily obtained from $10 \mu \mathrm{m}$ crystals and from $20 \mu \mathrm{m}$ crystals.

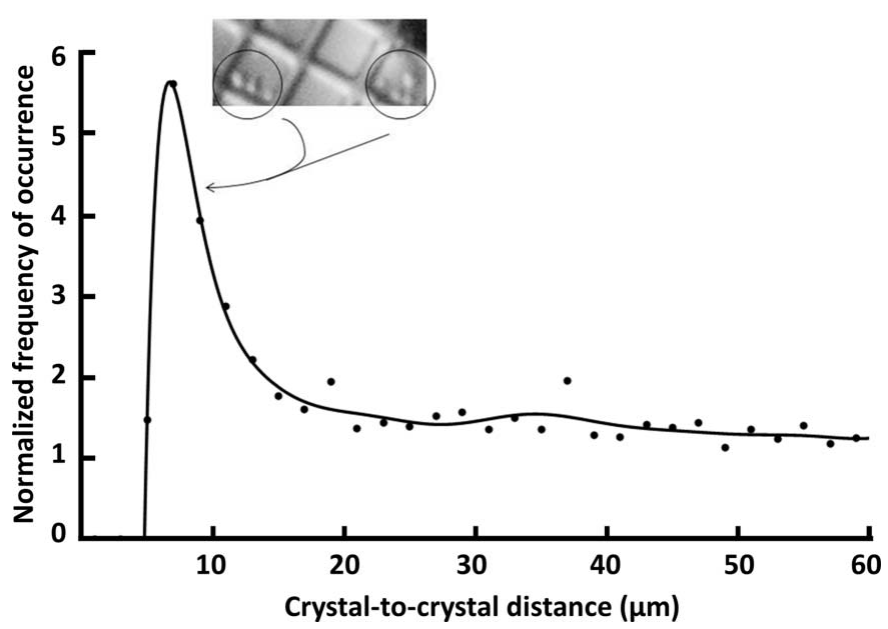

Figure 7

Severe clustering of insulin crystals made indexing difficult for $5 \mu \mathrm{m}$ crystals (seen here on a micro-mesh with $20 \mu \mathrm{m}$ openings). Frequently, our scanned images were either empty or had multiple overlapping diffraction patterns (inset). The attraction between crystals is predominantly short range. The $x, y$ coordinates were determined for 450 crystals visible on a $700 \mu \mathrm{m}$ micro-mesh. The distance between each crystal and every other crystal was calculated. The graph shows the relative probability of encountering neighbors as a function of distance $(\mu \mathrm{m})$. The curve is normalized by dividing the experimental probability distribution by the probability distribution obtained from computergenerated random locations; since the graph is normalized, a random experimental distribution would have a flat probability ratio equal to unity. A polynomial best fit is also shown. The spike at $7 \mu \mathrm{m}$ (slightly larger than the average crystal size) indicates that there are many crystals located adjacent to other crystals. We hypothesize that surface tension may exert a short-range attractive force that draws crystals together. The relative probability falls below unity for distances of $200 \mu \mathrm{m}$ or more. 
are serially exposed on a moving crystal conveyor belt (our method was analogous to recent serial crystallography applications performed on micro-meshes and in situ crystallization plates) (Ji et al., 2009; Axford et al., 2012). As has been reported by others, uncomfortably high $R_{\text {merge }}$ values are a natural consequence of merging data from multiple specimens, but this does not deter a high-quality molecular model. Aggressive efforts to minimize the solvent envelope around protein crystals can reduce the solvent contribution to background and improve the signal-to-noise of the X-ray diffraction data. We report two ways in which aggressive solvent minimization can induce crystals to arrange themselves in ways that negatively impact the data. First, crystals can aggregate into clusters, causing the X-ray beam to illuminate either too many crystals or none at all. Second, crystals can preferentially orient relative to the flat surface on which they are deposited (such as the flat micro-mesh, the smooth plate bottom or the conveyor belt). Preferential orientation of a crystallographic axis may cause serial crystallography experiments to over-sample one region of reciprocal space while neglecting other regions of reciprocal space.

Insulin crystals were observed to preferentially orient with the crystallographic $c$ axis normal to the surface that they are deposited on. In contrast, lysozyme crystals were not observed to preferentially orient. Preferentially oriented crystals may serially transit the X-ray beam aligned so that large regions of reciprocal space are unmeasurable. The effects of preferential orientation on completeness can be avoided by positioning the data-collection surface at an angle to the incident beam. Alternatively, a second data-collection pass may be used to reveal regions of reciprocal space that are forbidden when the support surface is initially normal to the X-ray beam. It is perhaps not surprising that flat insulin crystals would tend to lie on a planar surface, while the orientation of cuboidal lysozyme crystals would remain random. This intuitive understanding of the causes of preferential orientation may facilitate recognizing situations where the problem is likely to occur, and designing experiments from the outset to avoid unmeasured regions of reciprocal space.

Careful specimen preparation with attention to solvent minimization yielded signal-to-noise and resolution limits comparable with those obtained from large single crystals. However, wicking away the solvent increased the attraction between crystals. In the case of very small specimens $(\sim 5 \mu \mathrm{m})$ the aggregation was crippling and clean single-crystal diffraction patterns were virtually unobtainable when probing with a $20 \mu \mathrm{m}$ beam. Subsequent experimentation at APS-GM/ CA confirmed that clustering can be overcome by using an $\mathrm{X}$-ray beam with a size comparable with the specimen size. We are investigating software improvements to handle multiple overlapping diffraction patterns, as well as physical methods to immobilize specimens while excess solvent is removed (such as adding structure to the kapton belt on our conveyor crystal delivery system to help disperse crystal clusters).

Personnel for this study were recruited largely through the Science Undergraduate Laboratory Internships Program
(SULI), supported through the US Department of Energy, Office of Science, Office of Workforce Development for Teachers and Scientists (WDTS). Major ongoing financial support for serial crystallography applications was through the Brookhaven National Laboratory/US Department of Energy, Laboratory Directed Research and Development Grant 11008 and from the Offices of Biological and Environmental Research and of Basic Energy Sciences of the US Department of Energy, and from the National Center for Research Resources (P41RR012408) and the National Institute of General Medical Sciences (P41GM103473) of the National Institutes of Health. Data for this study were measured at beamline X25, X29, X12c and X12b of the National Synchrotron Light Source and at beamline 23ID-B of the Advanced Photon Source. Financial support comes principally from the Offices of Biological and Environmental Research and of Basic Energy Sciences of the US Department of Energy, and from the National Center for Research Resources of the National Institutes of Health. GM/CA CAT is funded in part with funds from the National Cancer Institute (Y1-CO1020) and the National Institute of General Medical Science (Y1-GM-1104). We thank Yasmin Nessim Samara for producing our YouTube videos and Dr Robert Fischetti for his kind support. Author contributions: ASS designed the experiment and wrote the paper. ASS, JDM and RMP grew crystals, obtained data and analyzed data. ASS, GSM, CGR, RJ, AMO and MA designed and built the labware. ASS, GSM and RMS trained and supervised student interns.

\section{References}

Axford, D. et al. (2012). Acta Cryst. D68, 592-600.

Boutet, S. \& Robinson, I. K. (2008). J. Synchrotron Rad. 15, 576-583. Brehm, W. \& Diederichs, K. (2014). Acta Cryst. D70, 101-109.

Cole, K. et al. (2014). PloS ONE, 9, e101036.

Cuttitta, C. M., Ericson, D. L., Scalia, A., Roessler, C. G., Teplitsky, E., Joshi, K., Campos, O., Agarwal, R., Allaire, M., Orville, A. M., Sweet, R. M. \& Soares, A. S. (2014). Acta Cryst. D. In the press. Diamond, R. (1974). J. Mol. Biol. 82, 371-391.

Diederichs, K. \& Karplus, P. A. (1997). Nat. Struct. Biol. 4, 269-275.

Ellson, R., Mutz, M., Browning, B., Lee, L., Miller, M. F. \& Papen, R. (2003). J. Assn. Lab. Autom. 8, 29-34.

Helliwell, J. R. (1988). J. Cryst. Growth, 90, 259-272.

Henderson, R. (1990). Proc. R. Soc. London B, 241, 6-8.

Héroux, A. et al. (2014). J. Synchrotron Rad. 21, 627-632.

Hodgson, K. O., Anderson, W. F., Berman, L. R., Fischetti, Hendrickson, W. A., Kirz, J., Makowski, L., Phillips, G. N., Smith, J. L., Sweet, R. M. \& Tsuruta, H. (2009). Workshop of the National Institutes of Health National Center for Research Resources and the National Institute of General Medical Sciences on Plans for Support of Future Life Science Synchrotron Research at NSLS-II, Final Report, 4-5 June 2009, Bethesda, MD, USA.

Holton, J. M. \& Frankel, K. A. (2010). Acta Cryst. D66, 393-408.

Incardona, M.-F., Bourenkov, G. P., Levik, K., Pieritz, R. A., Popov, A. N. \& Svensson, O. (2009). J. Synchrotron Rad. 16, 872-879.

Ji, X., Sutton, G., Evans, G., Axford, D., Owen, R. \& Stuart, D. I. (2010). EMBO J. 29, 505-514.

Kubalek, E. W., Kornberg, R. D. \& Darst, S. A. (1991). Ultramicroscopy, 35, 295-304.

Langer, G., Cohen, S. X., Lamzin, V. S. \& Perrakis, A. (2008). Nat. Protocols, 3, 1171-1179. 
Murshudov, G. N., Skubák, P., Lebedev, A. A., Pannu, N. S., Steiner, R. A., Nicholls, R. A., Winn, M. D., Long, F. \& Vagin, A. A. (2011). Acta Cryst. D67, 355-367.

Otwinowski, Z. \& Minor, W. (2001). DENZO and Scalepack. Crystallography of Biological Macromolecules 2. Dordrecht: Kluwer.

Roessler, C. G., Kuczewski, A., Stearns, R., Ellson, R., Olechno, J., Orville, A. M., Allaire, M., Soares, A. S. \& Héroux, A. (2013). J. Synchrotron Rad. 20, 805-808.

Skinner, J. M., Cowan, M., Buono, R., Nolan, W., Bosshard, H., Robinson, H. H., Héroux, A., Soares, A. S., Schneider, D. K. \& Sweet, R. M. (2006). Acta Cryst. D62, 1340-1347.

Soares, A. S., Caspar, D. L. D., Weckert, E., Héroux, A., Hölzer, K., Schroer, K., Zellner, J., Schneider, D., Nolan, W. \& Sweet, R. M. (2003). Acta Cryst. D59, 1716-1724.

Soares, A. S., Engel, M. A., Stearns, R., Datwani, S., Olechno, J., Ellson, R., Skinner, J. M., Allaire, M. \& Orville, A. M. (2011). Biochemistry, 50, 4399-4401.
Teng, T.-Y. \& Moffat, K. (2002). J. Synchrotron Rad. 9, 198-201.

Tsai, Y., McPhillips, S. E., González, A., McPhillips, T. M., Zinn, D., Cohen, A. E., Feese, M. D., Bushnell, D., Tiefenbrunn, T., Stout, C. D., Ludaescher, B., Hedman, B., Hodgson, K. O. \& Soltis, S. M. (2013). Acta Cryst. D69, 796-803.

Vagin, A. \& Teplyakov, A. (1997). J. Appl. Cryst. 30, 1022-1025.

Villaseñor, A. G., Wong, A., Shao, A., Garg, A., Donohue, T. J., Kuglstatter, A. \& Harris, S. F. (2012). Acta Cryst. D68, 893900.

Weierstall, U., Doak, R. B., Spence, J. C. H., Starodub, D., Shapiro, D., Kennedy, P. \& Chapman, H. N. (2008). Exp. Fluids, 44, 675689.

Yin, X., Scalia, A., Leroy, L., Cuttitta, C. M., Polizzo, G. M., Ericson, D. L., Roessler, C. G., Campos, O., Ma, M. Y., Agarwal, R., Jackimowicz, R., Allaire, M., Orville, A. M., Sweet, R. M. \& Soares, A. S. (2014). Acta Cryst. D70, 1177-1189.

Zhang, Z., Sauter, N. K., van den Bedem, H., Snell, G. \& Deacon, A. M. (2006). J. Appl. Cryst. 39, 112-119. 